\title{
Growth cartilage expression of growth hormone/insulin-like growth factor I axis in spontaneous and growth hormone induced catch-up growth
}

\author{
Iva Brito ${ }^{\text {a,b,*}, ~ H e l e n a ~ G i l-P e n ̃ a ~}{ }^{c}$, Inés Molinos ${ }^{c}$, Vanessa Loredo ${ }^{c}$, Tiago Henriques-Coelho ${ }^{\text {b,d }}$, \\ Alberto Caldas-Afonso ${ }^{\text {b,d }}{ }^{\text {, Fernando Santos }}{ }^{\mathrm{c}}$ \\ a Pediatric Rheumatology Unit, Pediatric Department, Hospital São João, Porto, Portugal \\ b Physiology and Cardiothoracic Surgery Department, Faculty of Medicine, Porto, Portugal \\ c Department of Pediatrics, Hospital Universitario Central de Asturias and University of Oviedo, Oviedo, Asturias, Spain \\ d Department of Pediatrics, Hospital São João, Porto, Portugal
}

\section{A R T I C L E I N F O}

\section{Article history:}

Received 20 June 2011

Received in revised form 21 December 2011

Accepted 19 April 2012

Available online 14 May 2012

\section{Keywords:}

Catch-up growth

Growth plate

Growth hormone

IGF-I

\begin{abstract}
A B S T R A C T
Introduction: Catch-up growth following the cessation of a growth inhibiting cause occurs in humans and animals. Although its underlying regulatory mechanisms are not well understood, current hypothesis confer an increasing importance to local factors intrinsic to the long bones' growth plate (GP).

Aim: The present study was designed to analyze the growth-hormone (GH)-insulin-like growth factor I (IGFI) axis in the epiphyseal cartilage of young rats exhibiting catch-up growth as well as to evaluate the effect of $\mathrm{GH}$ treatment on this process.

Material and methods: Female Sprague-Dawley rats were randomly grouped: controls (group C), 50\% diet restriction for 3 days + refeeding (group CR); 50\% diet restriction for 3 days + refeeding \& GH treatment (group CRGH). Analysis of GH receptor (GHR), IGF-I, IGF-I receptor (IGF-IR) and IGF binding protein 5 (IGFBP5) expressions by real-time PCR was performed in tibial growth plates extracted at the time of catch-up growth, identified by osseous front advance greater than that of $C$ animals.

Results: In the absence of GH treatment, catch-up growth was associated with increased IGF-I and IGFBP5 mRNA levels, without changes in GHR or IGF-IR. GH treatment maintained the overexpression of IGF-I mRNA and induced an important increase in IGF-IR expression.

Conclusions: Catch-up growth that happens after diet restriction might be related with a dual stimulating local effect of IGF-I in growth plate resulting from overexpression and increased bioavailability of IGF-I. GH treatment further enhanced expression of IGF-IR which likely resulted in a potentiation of local IGF-I actions. These findings point out to an important role of growth cartilage GH/IGF-I axis regulation in a rat model of catch-up growth.
\end{abstract}

(c) 2012 Elsevier Ltd. All rights reserved.

\section{Introduction}

Longitudinal growth may be impaired in several chronic disorders like inflammatory bowel diseases, juvenile idiopathic arthritis and malnutrition as well as by exposure to some drugs. The term catch-up growth, introduced by Prader et al. [1], describes the phenomenon by which longitudinal growth velocity transiently stands above the statistical limits of normality for age and/or maturity after the removal of a growth-inhibiting condition. Catch-up growth occurs in humans and animals and its underlying pathogenic mechanism is not clear [2,3]. A recent hypothesis proposes an intrinsic essential mechanism for catch-up growth in the long bones' growth plate [4].

Linear bone growth results from proliferation, hypertrophy and matrix production of cartilage cells in growth plate, on whose

\footnotetext{
* Corresponding author at: Pediatric Rheumatology Unit, Department of Pediatrics, Hospital São João, Porto, Portugal. Tel.: + 351932348073.

E-mail address: ivaobrito@hotmail.com (I. Brito).
}

metaphyseal end, bone replaces the cartilage [5]. Growth plate of endochondral bones is comprised of cells representing various states of differentiation: the resting zone, the proliferative zone with actively dividing cells, and the hypertrophic zone with maturing cells enlarging and ultimately dying [6]. These proliferative and maturation processes are induced by numerous systemic endocrine effectors such as circulating growth hormone $(\mathrm{GH})$. The effects of $\mathrm{GH}$ are to a great extent mediated by the actions of insulin-like growth factor-I (IGF-I), a peptide growth factor produced in great abundance by hepatic tissue, source of the circulating plasma IGF-I [7], but also locally produced by chondrocytes. Likewise, growth hormone receptor (GHR) [8-10] have been localized in several regions of the growth cartilage, demonstrating that chondrocytes are capable of responding directly to $\mathrm{GH}$ in a paracrine way. Cellular proliferation and apoptosis are carefully coordinated processes within the growth plate. The GH axis is known to play a critical role in proliferation and IGF-I is an identified regulator of apoptosis in many tissues $[11,12]$. The interplay of proliferation and apoptosis in the developing growth plate has only recently been 
investigated and has focused on the hypertrophic chondrocytes which are known to undergo apoptosis [13-18].

The regulation of longitudinal growth is influenced by numerous systemic and local factors. Although the interplay among these factors is still unclear [6], GH and IGF-I are crucial. Circulating GH can act directly on growth plate stimulating the local IGF-I synthesis, which in turns induces chondrocyte expansion [19]. Additionally, there are some evidences that systemic IGF-I could also stimulate growth plate chondrocytes [20]. However, locally derived IGF-I seems to be more important in longitudinal growth than systemic IGF-I [21]. Abnormalities of the $\mathrm{GH} / \mathrm{IGF}-\mathrm{I}$ axis are one of the major determinants of growth retardation in children with chronic illnesses [22]. There are experimental and clinical evidences demonstrating important alterations in GH/IGF-I axis during chronic diseases, like chronic renal failure [23], chronic bowel inflammatory diseases [24] and juvenile idiopathic arthritis [25]. It is well known that treatment with GH potentiates catch-up growth in several conditions [5,26]. The GH/IGF-I axis in the growth plate at the time of catch-up growth was previously implicated by Gat-Yablonski in a rodent model of food restriction [9]. Nevertheless, how catch-up growth is influenced by GH treatment is largely unknown.

A previous experimental study of our group [5] examined the pattern of catch-up growth in young rats after an inhibitory growth condition. The existence of a growth recovery implies the return to the previous growth path after noxious stimulus suppression. Our experimental model showed that growth rate acceleration inherent to a spontaneous catch-up, was associated with enhancement of proliferative activity, and specially, to an expansion of the hypertrophic zone of the cartilage. GH administration started at the time of noxious stimulus suppression, further accelerated catch-up growth velocity, this effect being likely related to a greater enlargement of the terminal chondrocyte height [5]. The study presented here used the above mentioned animal model to determine if the ability to exhibit catch-up growth and the effect of GH treatment on this process are related to alterations in the growth plate GH-IGF-I axis modulation.

\section{Materials and methods}

\subsection{Experimental protocol}

Samples from animals used in a formerly published study were utilized [5]. Briefly, young female Sprague-Dawley rats (Harlam Iberia, Barcelona, Spain) were acclimatized to the experimental area and randomly grouped as follows: controls fed ad libitum (group C), diet restriction for 3 days + refeeding (group CR), diet restriction for 3 days + refeeding $\&$ GH treatment (group CRGH). On days 11,12 and 13 of the protocol, groups CR y CRGH received 50\% of the average daily food consumed by the same animals the previous three days. CRGH animals were treated with $3.3 \mathrm{mg} / \mathrm{kg} /$ day recombinant human GH (Norditropin, Novo Nordisk Pharm, Madrid-Spain) given by intraperitoneal route from day $14 \mathrm{on}$. Untreated animals received the same volume of vehicle (saline). Rats were killed at different time points after refeeding ( $n=5$ rats each time). Catch-up growth, assessed by measurement of osseous front advance by calcein labeling, was found in CR and CRGH groups on day 21 (C: $164.5 \pm 7.7 \mu \mathrm{m}$; CR: $186.1 \pm 6.1 \mu \mathrm{m}$; CRGH: $201.1 \pm 4.4 \mu \mathrm{m})$. Therefore, samples extracted on day 21 were used for the study presented here. The study complied with current legislation on animal experiments in the European Union and was approved by our Institution Ethics Committee for Investigation with animals.

\subsection{Samples}

The proximal ends of both tibiae were obtained after sacrifice by exsanguination under anesthesia. The dissection of growth plate was performed with magnifying lens and a scalpel to allow removing the adjacent bone zones accurately. The right tibiae were immediately frozen in liquid nitrogen for mRNA studies and the left tibiae were embedded in methylmethacrylate, fixed in 4\% paraformaldehyde (PFA) and used for immunohistochemistry and in situ hybridization studies on growth plate as previously described [27-29].

\subsection{Immunohistochemistry}

Immunohistochemical staining was performed in PFA-fixed frontal sections ( $5 \mu \mathrm{m}$ thick) of proximal tibiae, using IGF-I and IGF binding protein 5 (IGFBP5) polyclonal antibodies (Santa Cruz Biotechnology Inc, CA, USA) as described elsewhere [27].

\subsection{In situ Hybridization}

IGF-I, GHR, IGF-I receptor (IGF-IR) and IGFBP-5 antisense and sense RNA probes were supplied by Genedect (Genedetect.com, Bradenton, FL, USA). The hybridization procedure was carried out as described elsewhere [27]. Two parallel sections served as negative controls. One section hybridized with a labeled sense riboprobe and a second section was incubated adding a negative control probe to the hybridization mixture. No hybridization signal was found in any of these negative controls.

\subsection{Real-time PCR}

mRNA was extracted from frozen tibiae samples using modified Chomczynski method [30]. RNA purity and integrity were assessed by 260/280 ratio measurement using an ultra violet spectrophotometer (Gene Quant Pro, Amersham Biosciences, GE Healthcare Spain) and an $1.5 \%$ agarose gel respectively. Samples were diluted to $1 \mathrm{mg} / \mathrm{ml}$, before reverse transcription, with a commercial kit (Qiagen Iberia, Madrid, Spain).

GHR, IGF-I, IGF-IR and IGFBP5 expressions were quantified by real-time PCR system (One-Step, Applied Biosystems, Foster City, CA, USA) using SYBR Green (ABgene Products, Thermo Fisher Scientific, Rockford, IL, USA) as fluorophore and $\beta$-actin as a housekeeping gene. For all the qPCR experiments, melting curve showed a unique product that corresponded with an exclusive temperature of DNA dissociation. Oligonucleotide primer pairs were as follows: for $\beta$-actin, forward: ACCAGGGTGTGATGGTGGGTAT and reverse: CGTCCCAGTTGACAATGC; for GHR, forward: AATTAATCCAAGCCTGAGGGAAA and reverse: GGAACGACACTTGGT GAATCG; for IGF-I, forward: TACCAGCTCGGCCACAGC and reverse: GTGGGCTTGTTGAAGTAAAAGC; for IGF-IR, forward: GCTGCTGGACCACAAATCG and reverse: TCGCTTCCCACACACACTTG; for IGFBP5, forward: GACCAAGGCCCCTGCCGCA and reverse: CACAGTTGGGCAGGTACACGGC. Primer sequences were designed with the Primer Express software (Perkin-Elmer Applied Biosystems) and synthesized by Invitrogen (Fisher-Invitrogen, Barcelona, Spain). Standard curves were obtained for each primer pair with efficiency near to 0.99 . Afterwards, qPCR analysis was performed by $\Delta \Delta \mathrm{Ct}$ values.

\subsection{Statistical analysis}

Results are given as mean \pm SEM and groups were compared using one-way ANOVA. When the normality test failed, ANOVA on ranks was used. When treatments were significantly different, Dunn's test and the Holm-Sidak method were used to perform pairwise multiple comparisons. Statistical significance was set at a twotailed value of $\mathrm{P}<0.05$.

\section{Results}

\section{1. $C$ and $C R$ groups}

\subsubsection{Immunohistochemical analysis}

Analysis of IGF-I and IGFBP5 by immunohistochemical technique showed no variations between $C$ and CR groups for the distribution pattern. The positive signals for both peptides were largely restrained to 
the hypertrophic zone (HZ) although IGF-I positive cells were found in the lower part of the proliferative zone (LPZ) only in the CR group.

\subsubsection{In situ hybridization assay}

The pattern of mRNA signaling distribution in $C$ and $C R$ groups for IGF-I, IGF-IR and IGFBP5 was similar to that described for the peptides. Positive cells were mostly present in the HZ stratum. Some cells sited in the LPZ were positive for IGF-I mRNA in the CR group (Fig. 1). GHR mRNA was located in the entire growth plate with no differences between groups (data not shown).

\subsubsection{Real time $P C R$}

Relative quantification of GHR and IGF-IR mRNA revealed no changes in CR group compared with $C$ animals. However, levels of IGF-I and IGFBP5 mRNAs were higher in CR than $C$ rats (Table 1 ).

\subsection{Effect of $G H$ treatment}

No variations were found in the distribution pattern observed by immunohistochemical or in situ hybridization assays for IGF-I, IGFIR, GHR or IGFBP5, compared with the CR group. However, GH treatment induced a significant overexpression of IGF-IR mRNA compared with CR group (Table 1) (Fig. 2).

\section{Discussion}

Several experimental models of catch-up growth have been described, differing in the timing and nature of the noxius stimulus for growth retardation. Food restriction is a widely used model in rodents. In the present study we used catch-up growth after diet restriction during 3 days. We previously demonstrated that this period of food restriction was associated with stimulated proliferation and greater hypertrophy of growth cartilage chondrocytes, as formerly described [16]. The present study characterizes local expression of GHR, IGF-I, IGF-IR, IGFBP5 during catch-up growth phenomenon and its modulation by growth hormone treatment. Moreover, the study offers some
Table 1

Relative fold change obtained by real time PCR for GHR, IGF-I, IGF-IR and IGFBP5 mRNAs in the tibial growth plate of the three groups of rats.

\begin{tabular}{lllll}
\hline & GHR & IGF-I & IGF-IR & IGFBP5 \\
\hline C & $1.00 \pm 0.176$ & $1.00 \pm 0.03$ & $1.00 \pm 0.25$ & $1.00 \pm 0.10$ \\
CR & $0.87 \pm 0.116$ & $2.32 \pm 0.78^{\alpha}$ & $0.85 \pm 0.28$ & $1.47 \pm 0.23^{\alpha}$ \\
CRGH & $0.87 \pm 0.102$ & $2.53 \pm 0.57^{\alpha}$ & $2.34 \pm 0.12^{\alpha, \beta}$ & $1.36 \pm 0.21^{\alpha}$ \\
\hline
\end{tabular}

Legend: C group - control animals; CR group - animals submitted to the noxious stimulus; CRGH group - animals submitted to the noxious stimulus treated with growth hormone.

GHR: Growth Hormone Receptor; IGF-I: Insulin-like Growth Factor-I; IGF-IR: Insulinlike Growth Factor-I Receptor; IGFBP5: Insulin-like Growth Factor Binding Protein 5. Data are mean \pm SEM. Superscripts $\alpha$ and $\beta$ mean significantly different $(P<0.05)$ from $\mathrm{C}$ and CR group, respectively.

insight into the molecular mechanisms underlying catch-up growth. Our findings indicate that IGF-I and IGFBP5 mRNAs were overexpressed in the tibial growth plates of the group of rats exhibiting catch-up growth. These results are in agreement with the findings from GatYablonski et al. that demonstrated that catch-up growth after 10 days of food restriction was associated with an increase in local protein levels of IGF-IR and in serum levels of IGF-I [9,31].

It has been described that IGFBP5, enhances IGF-I-driven chondrocyte proliferation, thereby better presenting it to its receptor [32]. Delivery of IGF-I from pools of IGF-I bound to low-affinity IGFBP-5 in extracellular matrix could potentiate the binding of ligand to the IGF-IR on the cell surface, enhancing the differentiation process mediated by the IGF-I. The observed coexistent elevation of IGFBP5 and IGFI likely facilitates bioavailability of local IGF-I [33] so that it can be hypothesized that stimulated paracrine and autocrine actions of IGFI are linked to the morphological and dynamic changes that take place in the chondrocytes at the time of catch-up growth and to the ensuing acceleration of longitudinal growth velocity.

The present study also shows that catch-up growth was not associated with significant modifications in the pattern of expression of $\mathrm{GH}$ IGF-I growth plate axis when assessed by immunohistochemistry or in

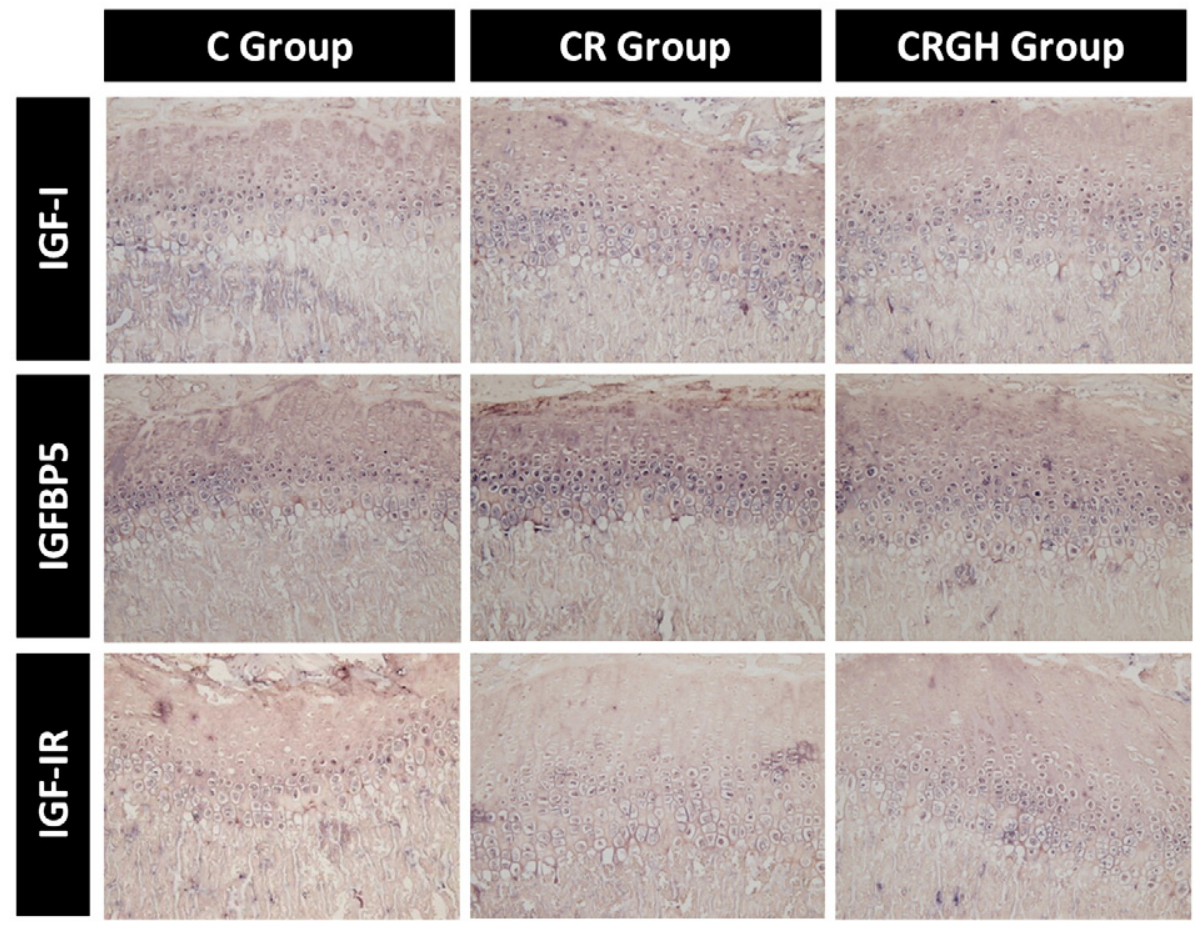

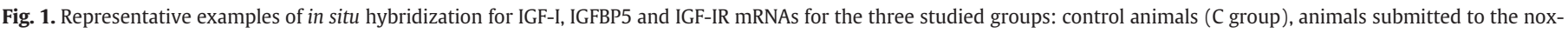

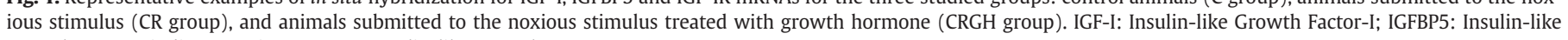
Growth Factor Binding Protein 5; IGF-IR: Insulin-like Growth Factor-I Receptor. 

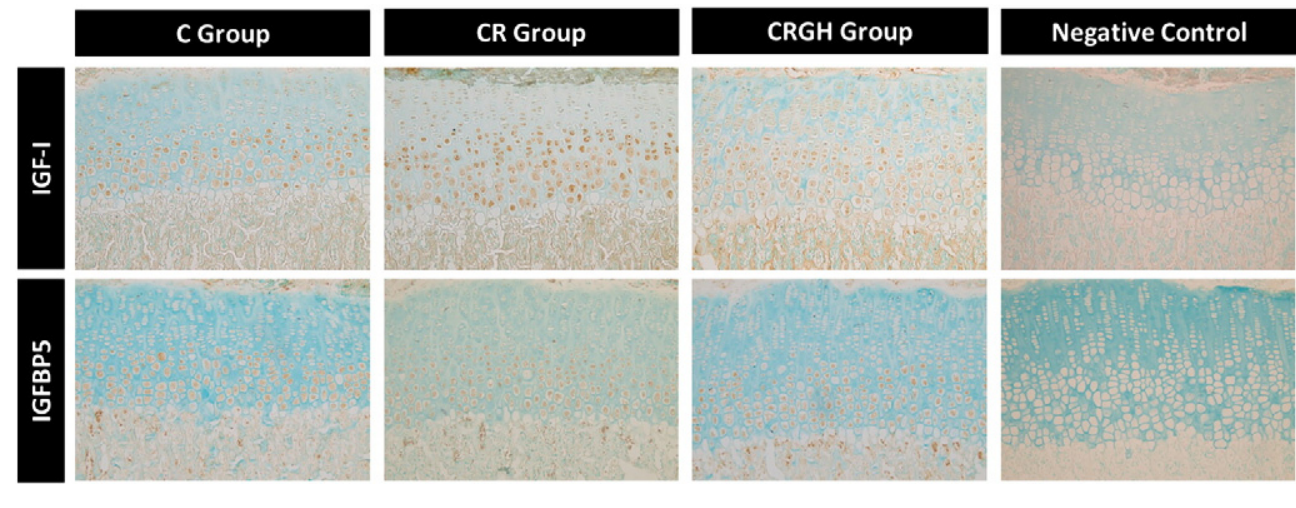

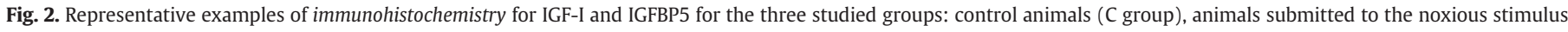

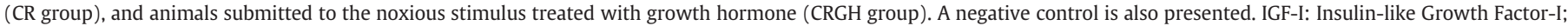
IGFBP5: Insulin-like Growth Factor Binding Protein 5.

situ hybridization. This lack of appreciable differences in the positive signaling between $\mathrm{C}$ and $\mathrm{CR}$ groups does not invalidate the quantitative changes detected by real time PCR because immunohistochemistry and in situ hybridization are good techniques for exploring changes in the spatial distribution of peptides and mRNAs, respectively, but not for measuring modifications in the magnitude of expression. For RNA extraction of the growth plate, we used manual microdissection. This is a simple and reliable method that minimized the contamination from surrounding tissues that can also express genes from GH/IGF-I axis [34].

Treatment with recombinant human GH has been widely used in several pediatric diseases associated with short stature and poor growth [35]. GH effects on epiphyseal cartilage chondrocyte proliferation are still controversial. GH can act directly on growth plate by increasing chondrocyte proliferation and stimulating local production of IGF-I [36]. In the present study we demonstrated that GH treatment did not modify the overexpression of IGF-I mRNA in the growth cartilage chondrocytes associated to the spontaneous catch-up growth of CR group. By contrast, we found that GH treatment induced an important increase in the growth plate IGF-IR expression These results reinforce the theory that therapy with GH potentiates catch-up growth by activating local IGF-I axis and support the crucial role of IGF-I locally produced in the long bones' growth plate in the postnatal growth [21].

The role of GH in the proliferation of epiphyseal cartilage chondrocytes is a matter of debate [37,38]. The microscopic examination of the in situ hybridization specimens suggested that the expression of IGF-I mRNA extended to the proliferative zone in the CR and CRGH groups. These findings together with the results of the real time PCR analysis indicate that the phenomenon of catch-up growth, either spontaneous or magnified by GH treatment, is associated with local stimulation of IGF-I in the growth plate. This is also in agreement with Isaksson et al. who demonstrated that local IGF-I amplification by GH stimulates chondrocyte proliferation and hypertrophy, contributing to bone growth. [19].

In summary, the present study shows for the first time that catchup growth that occurs after a short period of food restriction is associated with an increased expression of IGF-I and IGFBP5 in the long bones' growth plate. GH treatment preserves these changes and presumably potentiates local IGF-I actions by increasing IGF-IR expression in the chondrocytes.

\section{References}

[1] A. Prader, J.M. Tanner, H.G. von, Catch-up growth following illness or starvation. An example of developmental canalization in man, J. Pediatr. 62 (1963) 646-659.

[2] B. Boersma, J.M. Wit, Catch-up growth, Endocr. Rev. 18 (5) (1997) 646-661.

[3] C.E. Farnum, et al., Effect of short-term fasting on bone elongation rates: an analysis of catch-up growth in young male rats, Pediatr. Res. 53 (1) (2003) 33-41.

[4] J. Baron, et al., Catch-up growth after glucocorticoid excess: a mechanism intrinsic to the growth plate, Endocrinology 135 (4) (1994) 1367-1371.
[5] I. Molinos, et al., Catch-up growth follows an abnormal pattern in experimental renal insufficiency and growth hormone treatment normalizes it, Kidney Int. 70 (11) (2006) 1955-1961.

[6] B.C. van der Eerden, M. Karperien, J.M. Wit, Systemic and local regulation of the growth plate, Endocr. Rev. 24 (6) (2003) 782-801.

[7] D. Le Roith, et al., The somatomedin hypothesis: 2001, Endocr. Rev. 22 (1) (2001) 53-74.

[8] A.M. Oberbauer, R. Peng, Growth hormone and IGF-I stimulate cell function in distinct zones of the rat epiphyseal growth plate, Connect. Tissue Res. 31 (3) (1995) 189-195.

[9] G. Gat-Yablonski, et al., Nutrition-induced catch-up growth at the growth plate, J. Pediatr. Endocrinol. Metab. 21 (9) (2008) 879-893.

[10] G.A. Werther, et al., Identification of growth hormone receptors on human growth plate chondrocytes, Acta Paediatr. Suppl. 82 (Suppl. 391) (1993) 50-53.

[11] A. Grimberg, P. Cohen, Role of insulin-like growth factors and their binding proteins in growth control and carcinogenesis, J. Cell. Physiol. 183 (1) (2000) 1-9.

[12] S.A. Murray, et al., IGF-1 activates p21 to inhibit UV-induced cell death, Oncogene 22 (11) (2003) 1703-1711.

[13] D. Chrysis, et al., Apoptosis is developmentally regulated in rat growth plate, Endocrine 18 (3) (2002) 271-278.

[14] C.E. Farnum, N.J. Wilsman, Morphologic stages of the terminal hypertrophic chondrocyte of growth plate cartilage, Anat. Rec. 219 (3) (1987) 221-232.

[15] G.J. Gibson, W.J. Kohler, M.B. Schaffler, Chondrocyte apoptosis in endochondral ossification of chick sterna, Dev. Dyn. 203 (4) (1995) 468-476.

[16] D. Lewinson, M. Silbermann, Chondroclasts and endothelial cells collaborate in the process of cartilage resorption, Anat. Rec. 233 (4) (1992) 504-514.

[17] H.I. Roach, New aspects of endochondral ossification in the chick: chondrocyte apoptosis, bone formation by former chondrocytes, and acid phosphatase activity in the endochondral bone matrix, J. Bone Miner. Res. 12 (5) (1997) 795-805.

[18] M. Zenmyo, et al., Morphological and biochemical evidence for apoptosis in the terminal hypertrophic chondrocytes of the growth plate, J. Pathol. 180 (4) (1996) 430-433.

[19] O.G. Isaksson, J.O. Jansson, I.A. Gause, Growth hormone stimulates longitudinal bone growth directly, Science 216 (4551) (1982) 1237-1239.

[20] M. Reinecke, et al., Effect of growth hormone and insulin-like growth factor (IGF-I) on the expression of IGF-I messenger ribonucleic acid and peptide in rat tibial growth plate and articular chondrocytes in vivo, Endocrinology 141 (8) (2000) 2847-2853.

[21] S. Yakar, et al., Circulating levels of IGF-1 directly regulate bone growth and density, J. Clin. Invest. 110 (6) (2002) 771-781.

[22] F. De Luca, Impaired growth plate chondrogenesis in children with chronic illnesses, Pediatr. Res. 59 (5) (2006) 625-629.

[23] H. Gil-Pena, et al., Alterations of growth plate and abnormal insulin-like growth factor I metabolism in growth-retarded hypokalemic rats: effect of growth hormone treatment, Am. J. Physiol. Renal Physiol. 297 (3) (2009) F639-F645.

[24] A. Ballinger, Fundamental mechanisms of growth failure in inflammatory bowel disease, Horm. Res. 58 (Suppl. 1) (2002) 7-10.

[25] A. Tsatsoulis, et al., Study of growth hormone secretion and action in growth-retarded children with juvenile chronic arthritis (JCA), Growth Horm. IGF Res. 9 (2) (1999) 143-149.

[26] R. Crabbe, et al., Recombinant human growth hormone for children born small for gestational age: meta-analysis confirms the consistent dose-effect relationship on catch-up growth, J. Endocrinol. Invest. 31 (4) (2008) 346-351.

[27] O. Alvarez-Garcia, et al., Rapamycin induces growth retardation by disrupting angiogenesis in the growth plate, Kidney Int. 78 (6) (2010) 561-568.

[28] O. Alvarez-Garcia, et al., Rapamycin retards growth and causes marked alterations in the growth plate of young rats, Pediatr. Nephrol. 22 (7) (2007) 954-961.

[29] E. Carbajo, et al., Histologic and dynamic changes induced by chronic metabolic acidosis in the rat growth plate, J. Am. Soc. Nephrol. 12 (6) (2001) 1228-1234.

[30] P. Chomczynski, N. Sacchi, The single-step method of RNA isolation by acid guanidinium thiocyanate-phenol-chloroform extraction: twenty-something years on, Nat. Protoc. 1 (2) (2006) 581-585. 
[31] N. Even-Zohar, et al., Nutrition-induced catch-up growth increases hypoxia inducible factor 1alpha RNA levels in the growth plate, Bone 42 (3) (2008) 505-515.

[32] D. Kiepe, et al., Differential expression of IGF system components in proliferating vs. differentiating growth plate chondrocytes: the functional role of IGFBP-5, Am. J. Physiol. Endocrinol. Metab. 290 (2) (2006) E363-E371.

[33] J.I. Jones, D.R. Clemmons, Insulin-like growth factors and their binding proteins: biological actions, Endocr. Rev. 16 (1) (1995) 3-[34].

[34] E.A. Parker, et al., Spatial and temporal regulation of GH-IGF-related gene expression in growth plate cartilage, J. Endocrinol. 194 (1) (2007) 31-40.
[35] P.C. Hindmarsh, M.T. Dattani, Use of growth hormone in children, Nat. Clin. Pract. Endocrinol. Metab. 2 (5) (2006) 260-268.

[36] O. Nilsson, et al., Endocrine regulation of the growth plate, Horm. Res. 64 (4) (2005) 157-165.

[37] K. Madsen, et al., Growth hormone stimulates the proliferation of cultured chondrocytes from rabbit ear and rat rib growth cartilage, Nature 304 (5926) (1983) 545-547.

[38] M.R. Hutchison, M.H. Bassett, P.C. White, Insulin-like growth factor-I and fibroblast growth factor, but not growth hormone, affect growth plate chondrocyte proliferation, Endocrinology 148 (7) (2007) 3122-3130 